\title{
Etude expérimentale du transfert du cobalt 60 dans une chaine trophique marine benthique
}

\author{
C. Amiard-Triquet \& J. C. Amiard \\ Centre d'Etudes Nucléaires de Cadarache, Département de Protection, \\ Service de Recherches Toxicologiques et Ecologiques, \\ Laboratoire de Radioécologie Continentale; \\ Saint Paul Lez Durance, France
}

\begin{abstract}
Experimental study on the transfer of cobalt-60 in a marine benthic food chain. Experiments were carried out in order to quantify the transfer of ${ }^{60} \mathrm{Co}$ from the environment to various species of a benthic food chain. The food chain tested was composed of the diatom Navicula sp., the bivalve Scrobicularia plana, the shore crab Carcinus maenas, and the rat Rattus rattus. The diatoms take up large quantities of radiocobalt. The radioactivity accumulated (per unit weight) by the organisms studied decreases from one trophic level to the next. This result is particularly interesting from the point of sanitary protection. There is some biological control in ${ }^{\circ} \mathrm{Co}$ uptake by these species. For both, invertebrate animals and rat, the quantity of "Co assimilated is independent of the quantity ingested with food. Preferential sites of cobalt accumulation are liver and kidneys in the rat, and hepatopancreas in the two invertebrate species examined.
\end{abstract}

\section{INTRODUCTION}

Avec une période physique de 5,2 ans, le cobalt 60 , émetteur de rayonnements hautement énergétiques, est un isotope particulièrement important d'un point de vue de radioprotection. D'autre part, le cobalt stable présente une importance industrielle certaine (Morral, 1970) et le molysmologue est donc amené à étudier son devenir dans le milieu.

En tant que constituant de la vitamine $B_{12}$, le cobalt remplit un rôle vital chez la plupart des espèces animales (SMTH, 1962, 1965). De plus, il participe aux systèmes enzymatiques (ECOLE DE Roscoff, 1970). Le cheminement de cet élément dans les châ̂nes trophiques apparaît donc évident et il y a lieu d'en définir les modalités.

La connaissance du cycle des radionucléides dans les chaînes alimentaires marines est essentiel pour pouvoir: (a) prévoir les concentrations des radionucléides dans les produits marins comestibles; (b) estimer les doses d'irradiation auxquelles sont soumises les populations animales en milieu marin; (c) évaluer l'impact des organismes marins sur la redistribution des radionucléides dans l'environnement marin (Cross et al., 1973).

D'autre part, d'un point de vue technique, la contamination des chaînes alimentaires constitue un excellent test de pollution du milieu. C'est ainsi que Carey et al. 
(1966) ont pu mesurer des concentrations croissantes de zinc 65 chez un «depositfeeder», Ophiura sp., et son prédateur, un carnivore benthique de l'espèce Luidia folialata, alors que le zinc 65 n'était pas détectable dans le sédiment ò̀ vivaient ces animaux. Cette technique a été fructueusement utilisée par AUBERT et son équipe pour étudier la toxicité des polluants métalliques (AUBERT et al., 1969, 1972, 1974).

Nous nous sommes attachés à étudier la contamination d'une chaîne trophique existant effectivement dans le milieu naturel et caractéristique des faciès de vasière: (a) le producteur primaire est représenté par Navicula sp., une espèce de Diatomées formant «thalle» à la surface de la vase; (b) le consommateur primaire (herbivore) est un Mollusque de l'espèce Scrobicularia plana da Costa qui pratique à la fois le «deposit-feeding» et le "filter-feeding» (ROBERT, 1971); (c) le consommateur secondaire (carnivore) est un Crustacé, Carcinus maenas L. Cette espèce est utilisée par l'Homme pour la préparation de la soupe de poissons et des espèces voisines sont recherchées sur le plan gastronomique. (d) Dans le but d'évaluer le risque encouru par l'Homme consommant des produits marins contaminés, un aliment préparé à partir de ces Crabes a été fourni à des Rats (consommateur tertiaire).

ROBERT (1971 et communication orale) nous a signalé la présence de cette chaine marine en baie de Bourgneuf (Loire-Atlantique) et nous avons pu observé son fonctionnement dans l'estuaire de la Loire. Dans l'étang lagunaire du Prévost (Hérault), GUELoRget \& MrChel (1974) ont constaté l'abondance de Scrobicularia plana et de Carcinus maenas (variété méditerranéenne).

\section{PROTOCOLE EXPERIMENTAL}

Nous exposerons brièvement ce protocole qui a déjà été décrit partiellement dans une publication antérieure (AMIARD \& AMIARD-TRIQUET, 1975), sauf en ce qui concerne le consommateur final.

\section{Contamination des Navicules}

Le milieu de culture (eau de mer de la Méditerranée enrichie de sels minéraux) est divisé en unités de $500 \mathrm{ml}$ et contaminé par le cobalt 60 à raison de $10 \mu \mathrm{Ci} / 1$. Lorsque les cultures (stériles et monospécifiques) sont bien développées, le milieu contaminé est remplacé par une eau de mer «froide» pendant 16 à $48 \mathrm{~h}$. L'eau de mer est ensuite renouvelée et la radioactivité $A$ des Navicules est mesurée. Dans une expérience antérieure (AMIARd \& AmIARd-Triquet, 1975) nous avons déterminé que le facteur de concentration par rapport à l'eau variait de 266 à 396 ce qui correspond à une radioactivité par unité de poids de 2,2 à $3,2 \mu \mathrm{Ci} / \mathrm{g}$ (Tableau 1).

\section{Contamination des Scrobiculaires}

Dans chaque unité de culture sont introduites cinq Scrobiculaires, repérées individuellement, pour une période de $24 \mathrm{~h}$ appelée «repas». A la fin du repas, la 
Tableau 1

Accumulation du cobalt 60 par les différentes espèces

\begin{tabular}{|lc|}
\hline Espèces & Radioactivité $(\mathrm{nCi} / \mathrm{g})$ \\
\hline Navicula sp. & $2200-3200^{* *}$ \\
Scrobicularia plana & $40,5 *$ \\
Carcinus maenas & 12,6 \\
Rattus rattus & $0,03^{*+}$ \\
$*$ organismes parvenus à l'état d'équilibre. & \\
+ mesure effectuée avant la fin de la digestion. & \\
\hline
\end{tabular}

radioactivité $B$ des Navicules qui n'ont pas été consommées est mesurée ainsi que la radioactivité $C$ de l'eau surnageante résultant d'une légère décontamination des Navicules.

Deux groupes témoins de cinq Scrobiculaires ont été maintenus pendant cinq jours dans cette eau de décontamination. La quantité de ${ }^{60} \mathrm{Co}$ retenue par ces individus est négligeable devant celle accumulée par les Scrobiculaires ayant reçu de la nourriture contaminée.

Chaque lot de Scrobiculaires est ensuite replacé dans $500 \mathrm{ml}$ d'eau de mer pure de tout contaminant pendant 48 a $72 \mathrm{~h}$. A la fin de cette période, dite de digestion, la radioactivité des Scrobiculaires est mesurée individuellement et la somme des radioactivités des cinq spécimens de chaque lot est appelée $D$. Un tel protocole est nécessaire si l'on ne veut pas inclure dans cette mesure la quantité de cobalt 60 transitant dans la lumière du tube digestif.

La radioactivité $F$ des fèces éliminées pendant cette période est mesurée pour chaque lot ainsi que la radioactivité $G$ résultant de l'excrétion sous forme liquide.

Ces opérations ont été répétées deux fois par semaine pendant 3 à 6,5 semaines (6 à 13 repas).

\section{Contamination de Carcinus maenas}

La nourriture des Crabes est constituée par des Scrobiculaires ayant reçu six repas. Deux fois par semaine, chaque Crabe reçoit une Scrobiculaire de radioactivité A' (quantité fournie). Une partie du Mollusque est quelquefois négligée par le Crabe (radioactivité B'). La radioactivité totale de chaque Crabe est mesurée sur l'animal vivant immédiatement après le repas (D') et après un laps de temps, variant de 48 à $72 \mathrm{~h}$, pendant lequel s'effectue la digestion (cette dernière mesure est notée $\mathrm{G}^{\prime}$ ).

Comme pour l'espèce précédente, le cobalt 60 éliminé pendant cette période sous forme de fèces (radioactivité J') ou d'excrétats liquides (radioactivité K') est mesuré.

\section{Contamination $\mathrm{du} R$ a $\mathrm{t}$}

En ce qui concerne le transfert du cobalt des produits marins aux Mammifères, une première approche a été effectuée. L'expérience a porté sur un seul Rat, Rattus rattus Wistar AG de sexe femelle et de poids $230 \mathrm{~g}$. 
L'animal était placé dans une cage à métabolisme. Pendant 4 semaines il a reçu quotidiennement $15 \mathrm{~g}$ d'un aliment composé de $5 \mathrm{~g}$ des Carcinus maenas précédemment contaminés, séchés et réduits en poudre et de $10 \mathrm{~g}$ des granulés constituant son alimentation habituelle. Il a jêuné le dixième et le douzième jours de l'expérience. Durant les huit premiers repas, la radioactivité de la nourriture était environ de 18 $\mathrm{nCi} /$ repas puis, pour les dix-huit repas suivants, de $32 \mathrm{nCi} /$ repas environ.

Généralement, les fèces et les urines étaient recueillies quotidiennement. Les fèces et les urines émises du vendredi au dimanche étaient recueillies seulement le lundi; la radioactivité de ces échantillons était mesurée ainsi que la radioactivité de la nourriture non ingérée.

A la fin de l'expérience, le Rat a été sacrifié, disséqué et la radioactivité de ses différents organes a été mesurée.

\section{Mesures de radioactivité}

Toutes les mesures ont été effectuées en spectrométrie $\gamma$. Il a été tenu compte du rendement des systèmes de détection et du rendement géométrique des échantillons ce qui a permis d'exprimer toutes les mesures en $\mathrm{nCi}$.

\section{RESULTATS}

\section{Expression des résultats}

\section{Scrobicularia plana}

Le mode de calcul a été explicité dans le rapport d'une expérience préliminaire (Amtard \& Amiard-Triquet, 1975). Les notations adoptées pour les mesures de radioactivité sont identiques.

Une variable supplémentaire doit être définie, la quantité $\mathrm{E}$ de cobalt assimilée par repas

$$
E=D_{\text {[après n repas] }}-D_{\text {[après }(n-1) \text { repas] }}
$$

Dans ces conditions, l'expression de la somme $H$ devient:

$$
\mathrm{H}=\mathrm{B}+\mathrm{C}+\mathrm{E}+\mathrm{F}+\mathrm{G} \text {. }
$$

Nous remarquons (Tableau 2) que pour l'ensemble de six ou de treize repas, le rapport $\mathrm{I}=\mathrm{H} / \mathrm{A}$, théoriquement égal à $\mathrm{I}$, varie en pratique de 0,83 à 1 . Ce bilan est relativement satisfaisant étant données les incertitudes sur les mesures expérimentales. 


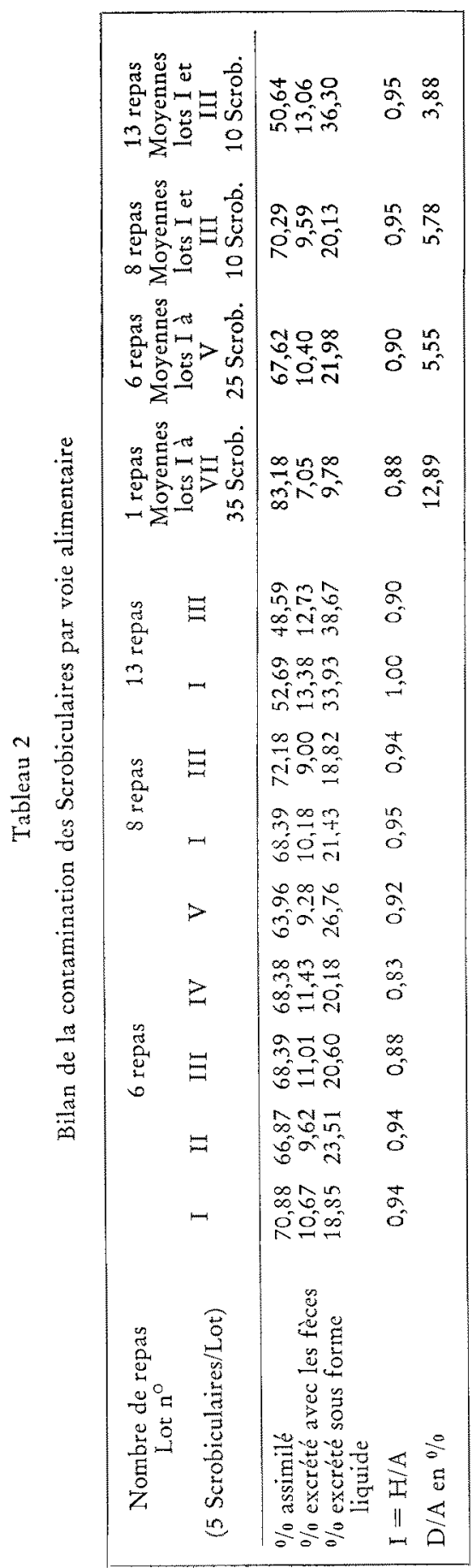




\section{Carcinus maenas}

Soit A' la radioactivité de la nourriture fournie au Crabe et B' la quantité de nourriture négligée par l'animal: théoriquement le Crabe ingère la quantité $C^{\prime}$ telle que:

$$
C^{\prime}=A^{\prime}-B^{3} \text {. }
$$

En fait, lors de la mastication de la nourriture, il se produit une perte de substance qui ne peut être mesurée directement et, en conséquence, la valeur C' constitue une surestimation de la quantité ingérée.

La quantité ingerérée réelle E' est donc déduite des comptages des Crabes «in totos:

$$
E^{3}=D^{\prime} \text { [après n repas] }-G^{\prime} \text { [après }(n-1) \text { repas et digestion] }
$$

Signalons que l'incertitude sur la mesure $\mathrm{D}$ ' et l'incertitude sur la mesure G' sont de l'ordre de 10 à $15 \%$.

La perte de nourriture qui se produit lors de la mastication peut être évaluée indirectement en comparant la quantité ingérée réelle $\mathrm{E}^{\prime}$ à la quantité ingérée théorique C'.

Le pourcentage ingéré sera calculé comme lc rapport:

$$
F^{\prime}=\frac{100 \cdot E^{\prime}}{A^{\prime}}=\frac{\text { Quantité ingérée réelle }}{\text { Quantité fournie }}
$$

La quantité de cobalt assimilée par le Crabe à la suite de chaque repas ( $\left.\mathrm{H}^{\prime}\right)$ sera calculée comme la différence:

$$
H^{\prime}=G^{\prime} \text { [après n repas et digestion] }-\mathrm{G}^{\prime} \text { [après }(\mathrm{n}-1) \text { repas et digestion] }
$$

Le pourcentage assimilé ou «rendement du repas» sera donc:

$$
I^{\prime}=\frac{100 \cdot G^{\prime}}{E^{\prime}}
$$

Les pourcentages excrérés sous formes de fèces ou d'excrétats liquides seront respectivement:

$$
\begin{gathered}
L^{\prime}=\frac{100 \cdot J^{\prime}}{E^{\prime}} \\
\text { et } M^{\prime}=\frac{100 \cdot K^{\prime}}{E^{\prime}}
\end{gathered}
$$

\section{Rattus rattus}

Connaissant la radioactivité de la nourriture fournie à chaque repas et celle de la nourriture négligée par le Rat, nous pouvons estimer par différence la quantité de ${ }^{60} \mathrm{Co}$ effectivement ingérée avec les aliments.

Connaissant les excrétions fécales et urinaires journalières, nous pouvons également estimer les quantités de ${ }^{60} \mathrm{Co}$ assimilées. 


\section{Résultats concernant Scrobicularia plana}

Nous constatons (Fig. 1 et 2) que la radioactivité des dix Scrobiculaires des lots I et III qui ont reçu treize repas n'augmente plus dès le huitième apport alimentaire. C'est donc qu'à ce stade, la quantité de cobalt 60 reçue avec la nourriture ingérée est égale à la quantité éliminée avec les fèces ou sous forme d'excrétats liquides. Nous

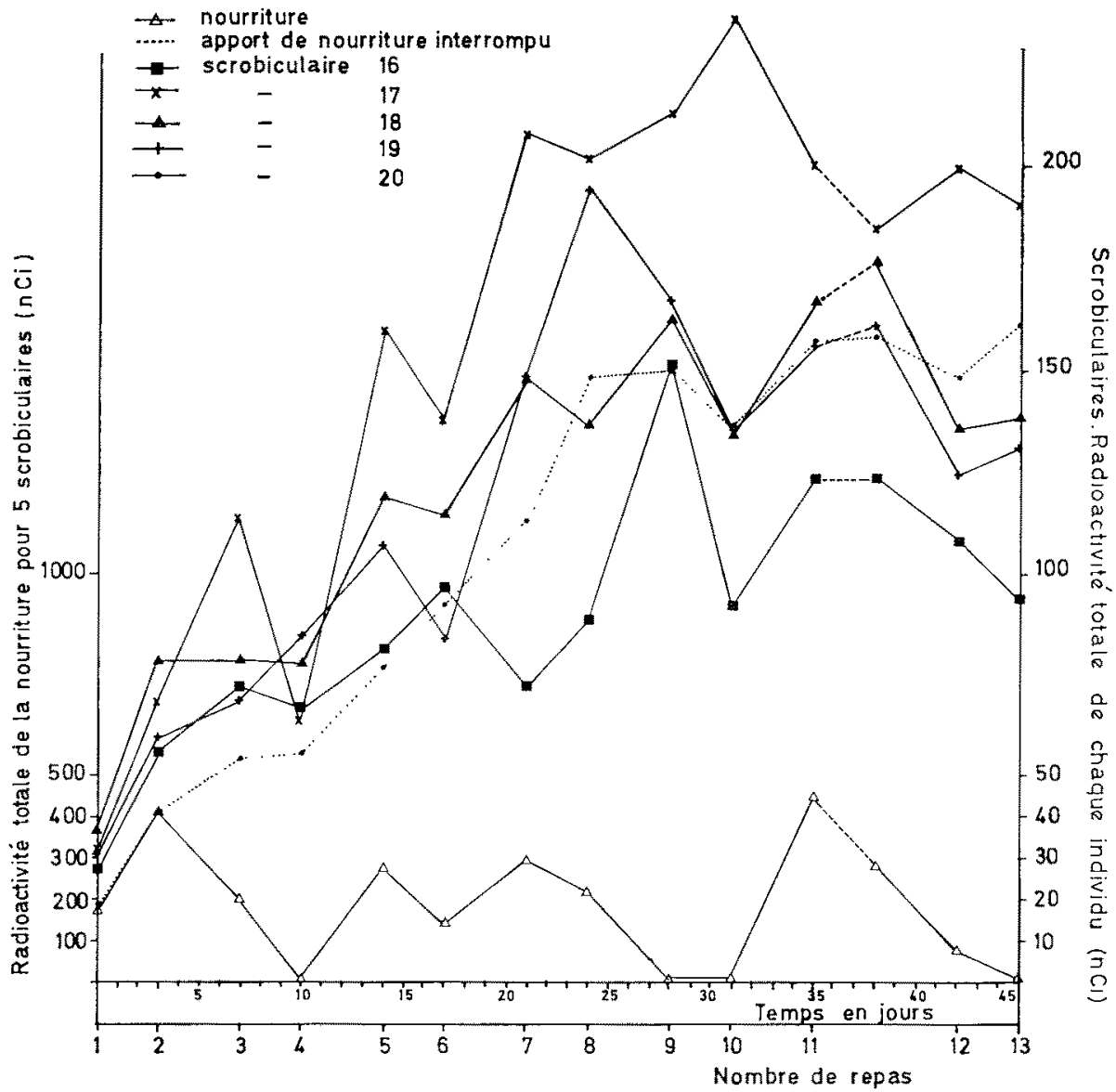

Fig. 1: Dynamique de la contamination de Scrobicularia plana par le cobalt 60: lot expérimental I

appellerons ce stade état d'équilibre. A ce moment, la radioactivité des Mollusques par unité de poids atteint $40,5 \mathrm{nCi} / \mathrm{g}$ (Tableau 1).

Les Figures 1 et 2 montrent que les fluctuations importantes de la radioactivité de la nourriture ingérée à chaque repas par les Scrobiculaires ne se reflètent pas sur les variations de la radioactivité des spécimens biologiques. Il semble donc que la 
quantité de cobalt 60 retenue par les Scrobiculaires n'est pas corrélée à la quantité présente dans la nourriture ingérée.

Le Tableau 2 nous donne les moyennes des pourcentages de cobalt 60 assimilé ou excrétés pour un repas (lots I à VII), pour l'ensemble des six premiers repas (lots I à V), de huit repas (lots I et III), de 13 repas (lots I et III) ainsi que les

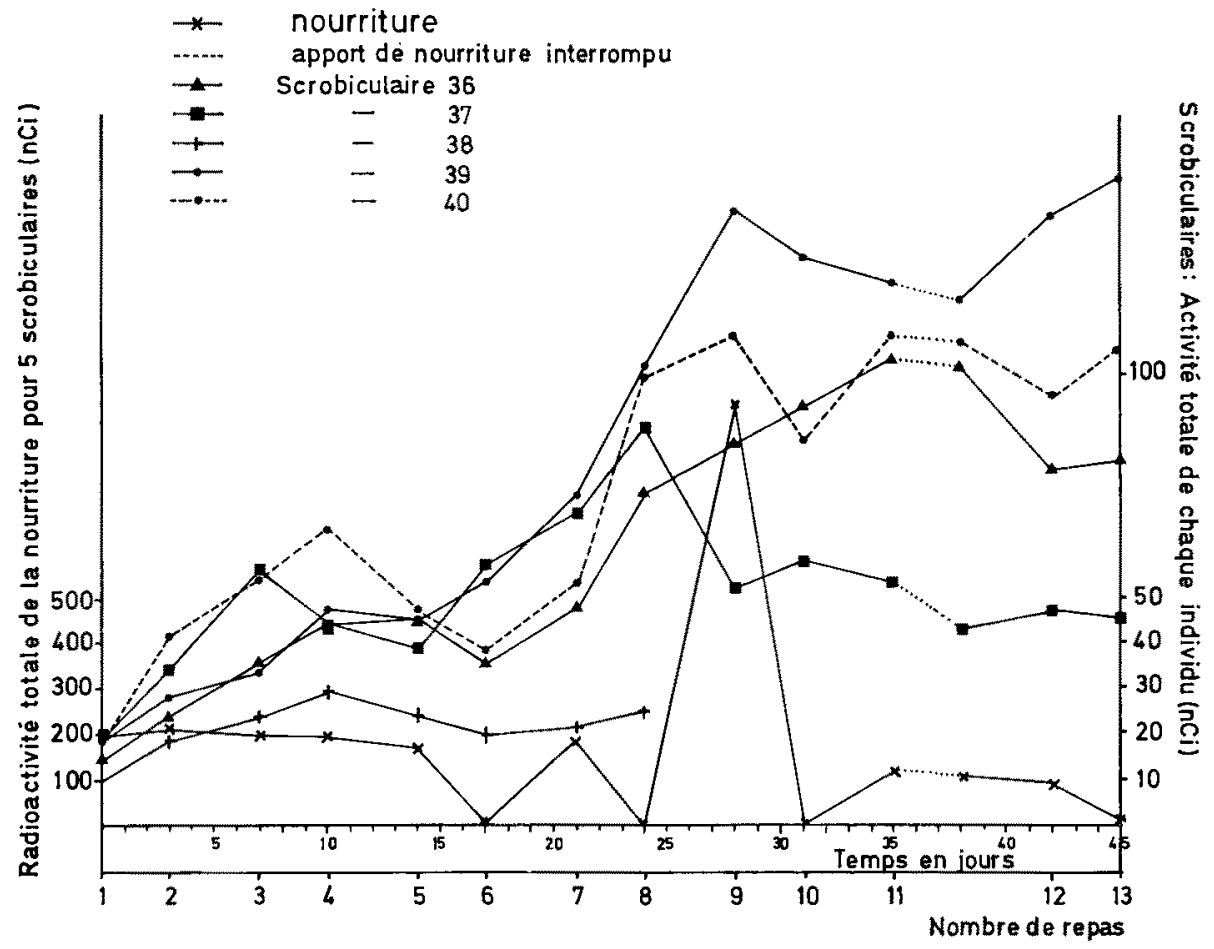

Fig. 2: Dynamique de la contamination de Scrobicularia plana par le cobalt 60: lot expérimental III

résultats détaillés lot par lot. Nous observons une décroissance du pourcentage de cobalt 60 assimilé en fonction du nombre de repas et, simultanément une augmentation des excrétions fécales et liquides.

Il est intéressant de constater que la radioactivité $\mathrm{D}$ des organismes après 1,6 , 8 ou 13 repas correspond à un pourcentage relativement faible de la quantité A de cobalt 60 mise à la disposition des Scrobiculaires $(12,89$ à $3,88 \%$ ).

\section{Résultats concernant Carcinus maenas}

Nous représentons dans les Figures 3 et 4 la dynamique de contamination des deux Crabes qui ont été maintenus le plus longtemps en expérience, $S_{2}$ et $S_{6}$. Nous 
constatons qu'après quatorze repas la radioactivité globale des animaux n'est pas encore stabilisée mais qu'elle augmente moins rapidement qu'au début de l'expérience. Il semble donc qu'à ce stade, on tende vers l'état d'équilibre. La radioactivité des Crabes par unité de poids atteint alors $12,6 \mathrm{nCi} / \mathrm{g}$ (Tableau 1).

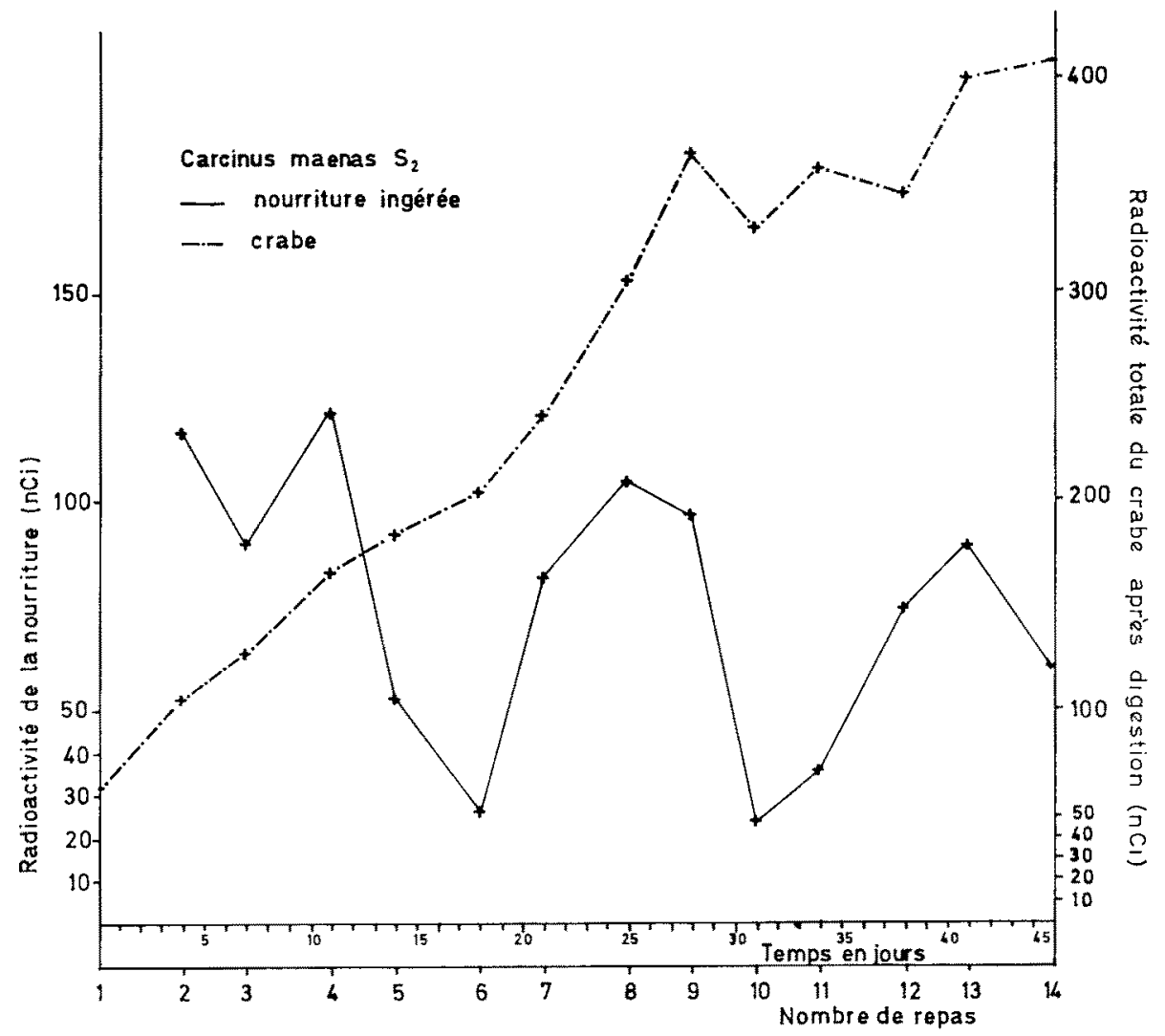

Fig. 3: Dynamique de la contamination de Carcinus maenas par le cobalt 60: crabe $S_{2}$

Les Figures 3 et 4 mettent en évidence que l'évolution de la contamination des Crabes ne suit pas les fluctuations de la radioactivité des proies ingérées.

La perte de nourriture qui se produit lors de la mastication atteint près de $40 \%$ de la quantité ingérée théorique $C^{\prime}$.

Le Tableau 3 nous donne les moyennes des pourcentages de cobalt 60 assimilé ou excrété pour un repas, et pour l'ensemble de neuf ou de quatorze repas. Comme pour l'espèce précédente, il se produit une décroissance du pourcentage de ${ }^{60} \mathrm{Co}$ assimilé en fonction du nombre de repas fournis aux animaux. A cette diminution de l'assimilation correspond une augmentation des deux types d'excrétion. L'excrétion liquide est toujours quantitativement plus importante que l'excrétion fécale.

Nous constatons (Tableau 3) que la somme des pourcentages assimilé et excrétés est toujours légèrement supérieure à $100 \%$. Ceci s'explique par le fait que 


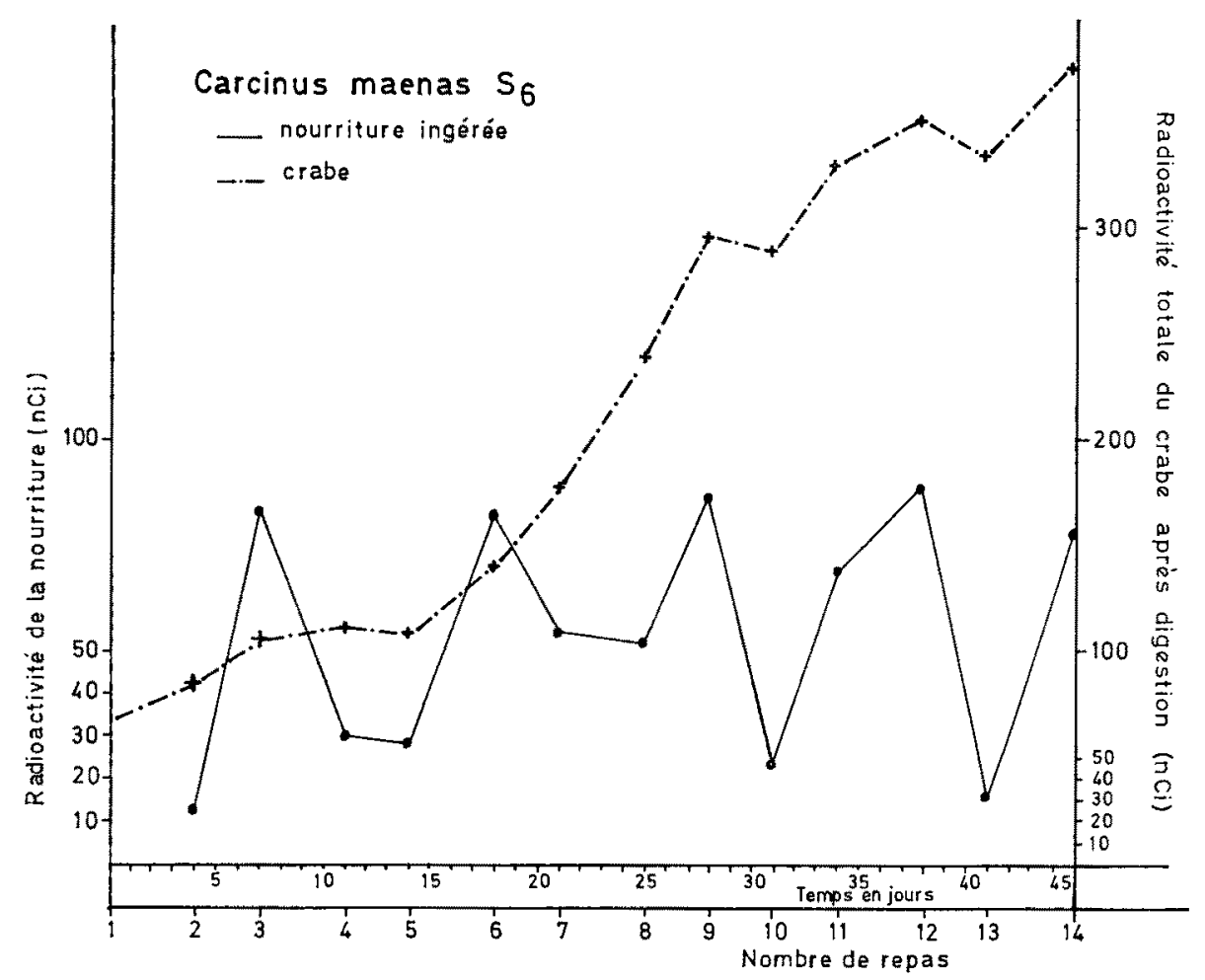

Fig. 4: Dynamique de la contamination de Carcinus maenas par le cobalt 60 : crabe $S_{6}$

dans l'établissement de ces pourcentages sont prises en compte des mesures expérimentales entachées d'incertitudes: 10 à $15 \%$ sur le comptage «in toto» des Crabes, 10 à $20 \%$ sur la mesure du cobalt 60 excrété sous forme liquide.

D'un point de vue pratique de radioprotection, nous remarquerons que la quantité de cobalt 60 assimilée par les Crabes ayant reçu huit à quatorze repas représente $39,30 \%$ de $A^{\prime}$, radioactivité de la nourriture mise à la disposition des animaux.

Tableau 3

Bilan de la contamination des Crabes par voie alimentaire

\begin{tabular}{|lcccc|}
\hline \multicolumn{1}{|c}{ Nombre de repas } & $\begin{array}{c}1 \text { repas } \\
(18 \text { crabes })\end{array}$ & $\begin{array}{c}9 \text { repas } \\
(7 \text { crabes })\end{array}$ & $\begin{array}{c}14 \text { repas } \\
(2 \text { crabes })\end{array}$ & $\begin{array}{c}\text { Moyennes } \\
9-14 \text { repas } \\
(9 \text { crabes })\end{array}$ \\
\hline \% assimilé & 98,72 & 80,74 & 74,30 & 79,31 \\
\% excrété avec les fèces & 3,59 & 4,50 & 6,95 & 5,05 \\
\% excrété sous forme & 6,56 & 19,38 & 38,15 & 23,55 \\
$\quad$ liquide & & 53,61 & 48,32 & 52,18 \\
\% ingéré & 39,45 & 39,75 & 39,52 \\
Perres dues à la mastication & & & & \\
$(\%)$ & & & \\
\hline
\end{tabular}




\section{Résultats concernant le Rat}

Cette première expérience nous a permis de constater que le Rat, qui pendant quatre semaines a reçu de la nourriture contaminée par ${ }^{60} \mathrm{Co}$, a une radioactivité de $7,5 \mathrm{nCi}$ soit $0,03 \mathrm{nCi} / \mathrm{g}$. A la dissection, 24 heures après la présentation du dernier repas, il apparait que l'estomac et l'intestin sont encore remplis de nourriture. Or, à eux deux, ces organes totalisent $6,3 \mathrm{nCi}$. La valeur $0,03 \mathrm{nCl} / \mathrm{g}$ de la radioactivité globale de l'animal constituc donc une importante surestimation de la quantité de cobalt 60 retenue dans l'organisme. Si l'on ne tient pas compte de la radioactivité présente dans le tube digestif, la quantité de cobalt 60 assimilée devient $0,005 \mathrm{nCi} / \mathrm{g}$.

La quantité totale de cobalt 60 présente dans le Rat à la dernière mesure correspond à $1,38 \%$ du cobalt 60 ingéré avec la nourriture en quatre semaines. Si, comme précédemment, on refait le calcul en ne tenant pas compte du cobalt 60 présent dans le tube digestif, le pourcentage assimilé est seulement de $0,22 \%$.

$98 \%$ du cobalt 60 sont éliminés avec les fèces et $2 \%$ seulement avec les urines. L'équilibre entre quantitées administrées et excrétées s'établit rapidement ( 2 à 4 jours) après les premiers repas ou après une augmentation de la radioactivité de la nourriture (9 9 me repas) (Fig. 5).

En dehors de l'estomac et de l'intestin, les reins et le foie sont les organes les plus contaminés.

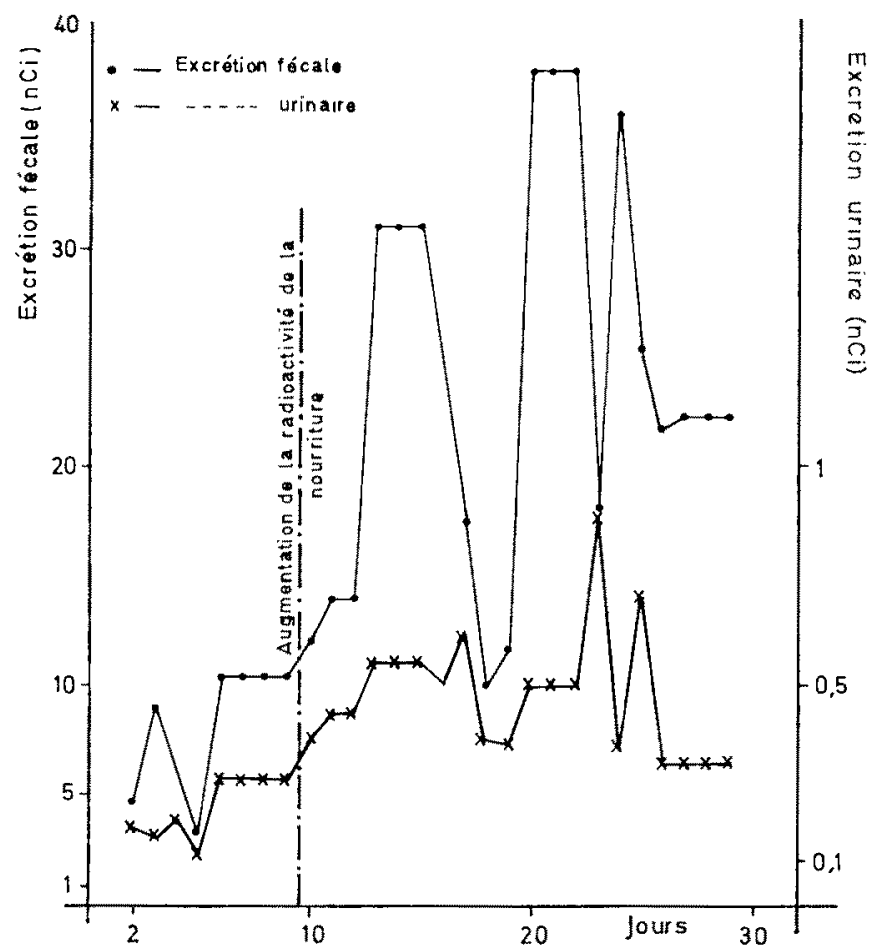

Fig. 5: Evolution des excrétions fécales et urinaires du Rat après ingestion répétées de cobalt 60 


\section{DISCUSSION}

Au niveau du producteur primaire, on observe une forte accumulation du cobalt 60. Ce résultat est en accord avec ceux observés «in situ» (Lowman, 1963) et s'explique en partie par le fait que dans les eaux de mer naturelles, le cobalt stable se trouve à une faible concentration. En Méditerranée, RoBERTSON (1970) a détecté des concentrations variant entre 4 et $43 \mathrm{ppb}$ (moyenne : 20). D'après FukaI (cité par Robertson, 1970), dans les eaux côtières de la région de Monaco, cette concentration est de l'ordre de 6 à $90 \mathrm{ppb}$ (moyenne : 30). Il en résulte une faible dilution isotopique et une radioactivité spécifique très élevée, tant pour le radiocobalt introduit dans le milieu sous forme d'effluent ou à la suite des explosions nucléaires dans l'atmosphère (CHIPMAN, 1966) que pour le cobalt 60 introduit expérimentalement dans nos milieux de culture. Nous avons utilisé une source d'activité spécifique $31,2 \mathrm{mCi} / \mathrm{mg}$ entraînant une surcharge du milieu en cobalt (stable + radioactif) de 320 ppb, c'està-dire que nous avons augmenté la concentration naturelle environ d'un facteur 10 .

Le Tableau 1 fait apparaître une diminution de la radioactivité des organismes par unité de poids lorsque l'on passe du producteur primaire au consommateur primaire, Scrobicularia plana, du consommateur primaire au consommateur secondaire, Carcinus maenas et au consommateur final, Rattus rattus. Il est intéressant de constater que ces résultats sont en parfait accord avec les études «in situ» effectuées en milieu marin (Lowman, 1963) ou en eau douce (Quierraza et al., 1969; Ophel \& Fraser, 1971).

Malgré les inconvénients liés à l'expérimentation (animaux stressés, volumes réduits, évolution des formes physicochimiques du cobalt pouvant être différente de ce qu'elle est en milieu marin) nous pouvons donc accorder un certain crédit à nos résultats. D'autre part, il faut signaler que nos animaux d'élevage se sont maintenus en parfaite santé pendant plus de trois mois: les Scrobiculaires ont constamment filtré l'eau de leur milieu et les Crabes ont toujours bien accepté la nourriture qui leur était proposée. De plus, les résultats obtenus pour les divers individus ou pour les différents lots ont présenté une homogénéité remarquable.

L'analyse des courbes (Fig. 1 à 4), avons nous signalé, montre que pour les deux espèces d'Invertébrés marins, la radioactivité des proies ingérées a peu d'influence sur l'évolution de la contamination de l'organisme. Ce résultat est tout à fait comparable à ceux obtenus antérieurement (AMIARD-TrRiquet \& AMIARD, 1974) sur des Carcinus maenas ayant ingéré des Arénicoles contaminées par le cobalt 60. Par contre, chez ces derniers, le pourcentage de ${ }^{60} \mathrm{Co}$ assimilé est un peu moins élevé que celui calculé pour l'ensemble de l'expérience chez les crabes $S_{9}$ et $S_{6}$ (c'est-à-dire les deux spécimens les plus proches de l'état d'équilibre). Ceci s'explique: d'une part, parce que dans l'expérience précédente, il s'agissait d'individus qui étaient effectivement à l'état d'équilibre; d'autre part, quelques modifications ont été apportées au protocole expérimental (température, nature et mode d'administration de la nourriture). Dans les deux expériences, l'excrétion liquide est toujours quantitativement plus importante que l'excrétion fécale.

Le phénomène est inverse chez le Rat ainsi que nous l'avons montré sur un seul individu mais comme le confirme SMITH (1962). Cet auteur rapporte en effet qu'après 
une administration orale unique de cobalt radioactif, $80 \%$ de la radioactivité sont retrouvés dans les fèces et $10 \%$ dans les urines.

Lorsque la quantité de ${ }^{60} \mathrm{Co}$ fournie avec la nourriture augmente (9ème repas), les excrétions fécales et urinaires augmentent parallèlement (Fig. 5). C'est dire que la quantité de cobalt 60 assimilée n'est pas proportionnelle à la quantité ingérée. L'établissement rapide d'un équilibre entre quantité ingérée et quantités excrétées avait déjà été observé par ABruniNA (1963).

Une publication antérieure (AMIARD-TRIQUET \& AMIARD, 1975) a montré que, quel que soit la nourriture fournie au Crabe (Arénicole ou Scrobiculaire), la répartition du cobalt dans l'organisme est identique. L'hépatopancréas et l'hémolymphe accumulent préférentiellement cet élément. Chez la Scrobiculaire, c'est également dans l'hépatopancréas que le cobalt 60 est essentiellement stocké. Nos résultats sur le Rat ainsi que les données de la littérature pour cette espèce et pour de nombreux autres Mammifères (SMITH, 1962; UNDERWOOD, 1971) mettent en évidence une localisation privilégiée du cobalt dans le foie et les reins.

\section{CONCLUSION}

Cette étude expérimentale* du transfert du cobalt 60 dans une chaîne trophique marine benthique et la littérature que nous avons passée en revue, nous permettent de remarquer un certain nombre de faits saillants:

(a) «In situ» et au laboratoire, le phytoplancton accumule des quantités importantes de radiocobalt.

(b) La radioactivité (rapportée à l'unité de poids) des organismes diminue lorsque l'on monte dans la pyramide de productivité: à une contamination élevée des producteurs correspondent des concentrations plus faibles chez les consommateurs primaires, plus faibles encore chez les consommateurs secondaires et tertiaires. Ce résultat est particulièrement intéressant dans une optique de protection sanitaire.

(c) D'un point de vue physiologique, nous retiendrons que le cobalt s'accumule préférentiellement dans des organes similaires chez les différentes espèces: hépatopancréas des Scrobiculaires et des Crabes, foie et reins des Mammifères.

(d) Nous avons observé que l'évolution des quantités assimilées par les Invertébrés étudiés et le Rat ne suit pas les fluctuations de la radioactivité des aliments. Nous pouvons en conclure que l'accumulation du cobalt par ces organismes n'est pas un phénomène passif et que l'assimilation de cet élément donne lieu à un contrôle biologique dont nous ne pouvons préciser ni la nature ni le site dans l'état actuel de nos recherches.

\section{RESUME}

1. Les Diatomées benthiques, Navicula sp., qui constituent le niveau trophique I de cette chaîne alimentaire expérimentale, accumulent des quantités importantes de cobalt 60 .

* Une publication ultérieure envisagera les résultats du transfert du cobalt 60 au long de cette chaîne alimentaire marine jusqu'au Mammifère d'un point de vue protection sanitaire. 
2. Les concentrations du radiocobalt dans les organismes des niveaux trophiques II (Scrobicularia plana), III (Carcinus maenas) et IV (Rattus rattus), diminuent vers le sommet de la pyramide de productivité qui, dans notre expérience, coïncide avec l'espèce la plus évoluée du point de vue de la classification zoologique. Soulignons l'intérêt de ce résultat dans une optique de protection sanitaire.

3. L'assimilation du cobalt donne lieu à un phénomène de régulation qui semble d'autant plus efficace que l'espèce considérée se situe plus haut dans l'échelle zoologique.

4. Le cobalt s'accumule essentiellement dans des organes similaires chez les différentes espèces animales: hépatopancréas des Scrobiculaires et des Crabes, foie et reins des Mammifères.

Remerciements. Nous remercions J. M. RoBert (Laboratoire de Physiologie Végétale, Université de Nantes) qui, ayant isolé la Diatomée utilisée comme producteur primaire dans cette expérience, a bien voulu nous en fournir des souches et nous indiquer les techniques de culture. Mm. Guelorget et Michel (Laboratoire d'Hydrobiologie, Université des Sciences et Technique du Languedoc, Montpellier) nous ont procuré en abondance Scrobiculaires et Crabes; qu'ils en soient remerciés.

\section{LITTERATURE CITEE}

Arrunina, G. A., 1963. Accumulation and excretion of ${ }^{\circ 0} \mathrm{Co}$ in animals and tissue doses during daily oral administration. In: The toxicology of radioactive substances. Ed. by A. A. Letavet \& E. B. Kurlyandskaya. Pergamon Press, New York 2, 30-43.

Amiard, J.-C. \& Amiard-Triquet, C., 1975. Expérience préliminaire à l'utilisation d'une châ̂ne trophique marine dans l'étude d'une pollution par le cobalt 60: bilan après une ingestion unique. Wat. Air. Soil Pollut. (sous presse).

Amiard-Triquet, C. \& Amiard, J.-C., 1974. Contamination de chaînes trophiques marines par le cobalt 60. Revue int. Océanogr. méd. 33, 49-59.

- 1975. Etude de l'organotropisme du cobalt 60 chez un Lamellibranche (Scrobicularia plana DA COSTA) et un Crustacé (Carcinus maenas L.) en fonction du vecteur de contamination. Oikos (sous presse).

Aubert, M., 1972. Pollutions chimiques et chaînes trophodynamiques marines. Revue int. Océanogr. méd. 28, 9-25.

- Bittel, R., Laumond, F., Romeo, M., Donnier, B. \& Barelli, M., 1972. Utilisation d'une chaîne trophodynamique de type pélagique pour l'étude des transferts des pollutions métalliques. Revue int. Océanogr. méd. 28, 27-52.

_ _ _ _ - 1974. Utilisation d'une chaîne trophodynamique de type néritique à Mollusque pour l'étude des transferts des polluants métalliques. Revue int. Océanogr. méd. 33, 7-29.

- Charra, R. \& Malaga, G., 1969. Etude de la toxicité des produits chimiques vis à vis de la chaîne biologique marine. Revue int. Océanogr. méd. 13-14, 45-72.

Carey, A. G., Pearcy, W. G. \& Osterberg, C. L., 1966. Artificial radionuclides in organisms in the Northeast Pacific off Oregon. In: Disposal of Radioactive Wastes into Seas, Oceans and Surface Waters. I.A.E.A., Vienna, 303-319.

Chipman, W. A., 1966. Food chains in the sea. In: Radioactivity and human diet. Pergamon Press, New York, 421-453.

Cross, F. A., Renfro, W. C. \& Gilat, E., 1973. A review of methodology for studying the transfer of radionuclides in marine food chains. I.A.E.A. working paper on reference methods for marine radiobiological studies, Monaco $61 \mathrm{pp}$. 
ECOLE D'ETE DE ROSCOFF, 1971. Interactions entre cations métalliques et macromolécules biologiques. Centre de la Recherche Scient, Paris, $144 \mathrm{pp}$.

Guelorget, O. \& Michel, P., 1974. Premières données quantitatives sur les peuplements benthiques de l'étang du Prévost, importance des Vénéridés. Assemblée générale de l'Union des Océanographes de France, Montpellier 6 (2), 15.

Lowman, F., 1963. Iron and cobalt in ecology. In: Radioecology. Ed. by. V. Schultz \& A. W. KLEMENT. Reinhold, New York, 561-568.

Morral, F. R., 1970. Developing metal: Cobalt. Materials technology. I. Am. Soc. Mech. Eng., New York, 472-479.

Ophel, I. L. \& Fraser, C. D., 1971. The fate of cobalt-60 in a natural freshwater ecosystem. In: Radionuclides in ecosystems. Ed. by D. J. Nel.son. Proc. 3rd nat. Symp. radioecology (Oak Ridge, Tenn.) 1, 323-327 (Conf.-710501-P1).

Queirraza, G., Smedile, E. \& Tibaldi, E., 1969. Sul transferimento di alcuni radionuclidi attraverso gli anelli di una catena alimentare fluviale. Atti Accad. naz. Lincei Rc. (Sci. fis. mat. nat.) 46, 81-90.

Robert, J.-M., 1971. Etude d'un faciès à Scrobiculaires et aspects divers de la biologie de ce Mollusque. Rapport de D.E.A., Université de Nantes.

Robertson, D. E., 1970. The distribution of cobalt in oceanic waters. Geochim. cosmochim. Acta 34, 553-567.

Smith, E. L., 1962. Cobalt. In: Mineral metabolism. Ed. by C. L. Comar \& F. Bronner. Acad. Pr., New York 2B, 349-369.

- 1965. Vitamin B12. Methuen, London, 180 pp.

Undfr woon, E. J., 1971. Trace elements in human and animal nutrition. Acad. Pr., New York, 141-169.

Adresse du premier auteur: Dr. C. Amiard-Triquet

Commissariat à l'Energic Atomique

Département de Protection

Service de Recherches Toxicologiques et Ecologiques

F-92260 Fontenay-aux-Roses

France 